\title{
SPECIAL FORMS OF GENERALIZED INVERSES OF ROW BLOCK MATRICES*
}

\author{
YONGGE TIAN ${ }^{\dagger}$
}

\section{Abstract.}

Given a row block matrix $[A, B]$, this paper investigates the relations between the generalized inverse $[A, B]^{-}$and the column block matrix $\left[\begin{array}{l}A^{-} \\ B^{-}\end{array}\right]$consisting of two generalized inverses $A^{-}$ and $B^{-}$. The first step of the investigation is to establish a formula for the minimal rank of the difference $[A, B]^{-}-\left[\begin{array}{l}A^{-} \\ B^{-}\end{array}\right]$, the second step is to find a necessary and sufficient condition for $[A, B]^{-}=\left[\begin{array}{c}A^{-} \\ B^{-}\end{array}\right]$to hold by letting the minimal rank be zero. Seven types of generalized inverses of matrices are taken into account.

Key words. Block matrix, Generalized inverse, Minimal rank formula, Moore-Penrose inverse, Schur complement.

AMS subject classifications. 15A03, 15A09.

1. Introduction. Let $\mathbb{C}^{m \times n}$ denote the set of all $m \times n$ matrices over the field of complex numbers. The symbols $A^{*}, r(A)$ and $\mathscr{R}(A)$ stand for the conjugate transpose, the rank, the range (column space) and the kernel of a matrix $A \in \mathbb{C}^{m \times n}$, respectively; $[A, B]$ denotes a row block matrix consisting of $A$ and $B$.

For a matrix $A \in \mathbb{C}^{m \times n}$, the Moore-Penrose inverse of $A$, denoted by $A^{\dagger}$, is the unique matrix $X \in \mathbb{C}^{n \times m}$ satisfying the four Penrose equations

(i) $A X A=A$,

(ii) $X A X=X$,

(iii) $(A X)^{*}=A X$,

(iv) $(X A)^{*}=X A$.

For simplicity, let $E_{A}=I_{m}-A A^{\dagger}$ and $F_{A}=I_{n}-A^{\dagger} A$. Moreover, a matrix $X$ is called an $\{i, \ldots, j\}$-inverse of $A$, denoted by $A^{(i, \ldots, j)}$, if it satisfies the $i, \ldots, j$ th equations. The collection of all $\{i, \ldots, j\}$-inverses of $A$ is denoted by $\left\{A^{(i, \ldots, j)}\right\}$. In particular, $\{1\}$-inverse of $A$ is often denoted by $A^{-}$. The seven frequently used generalized inverses of $A$ are $A^{(1)}, A^{(1,2)}, A^{(1,3)}, A^{(1,4)}, A^{(1,2,3)}, A^{(1,2,4)}$ and $A^{(1,3,4)}$, which have been studied by many authors; see, e.g., $[1,2,5,11]$. The general expressions of the seven generalized inverses of $A$ can be written as

$$
\begin{aligned}
A^{-} & =A^{\dagger}+F_{A} V+W E_{A}, \\
A^{(1,2)} & =\left(A^{\dagger}+F_{A} V\right) A\left(A^{\dagger}+W E_{A}\right), \\
A^{(1,3)} & =A^{\dagger}+F_{A} V,
\end{aligned}
$$

* Received by the editors 3 August 2005. Accepted for publication 30 October 2005. Handling Editor: Ravindra B. Bapat.

${ }^{\dagger}$ School of Economics, Shanghai University of Finance and Economics, Shanghai 200433, China (yongge@mail.shufe.edu.cn). 


$$
\begin{gathered}
\text { Y. Tian } \\
A^{(1,4)}=A^{\dagger}+W E_{A}, \\
A^{(1,2,3)}=A^{\dagger}+F_{A} V A A^{\dagger}, \\
A^{(1,2,4)}=A^{\dagger}+A^{\dagger} A W E_{A}, \\
A^{(1,3,4)}=A^{\dagger}+F_{A} V E_{A},
\end{gathered}
$$

where the two matrices $V$ and $W$ are arbitrary; see [1]. Obviously,

$$
\begin{aligned}
& \left\{A^{(1,3,4)}\right\} \subseteq\left\{A^{(1,3)}\right\} \subseteq\left\{A^{-}\right\}, \quad\left\{A^{(1,3,4)}\right\} \subseteq\left\{A^{(1,4)}\right\} \subseteq\left\{A^{-}\right\} \\
& \left\{A^{(1,2,3)}\right\} \subseteq\left\{A^{(1,2)}\right\} \subseteq\left\{A^{-}\right\}, \quad\left\{A^{(1,2,4)}\right\} \subseteq\left\{A^{(1,2)}\right\} \subseteq\left\{A^{-}\right\} \\
& \left\{A^{(1,2,3)}\right\} \subseteq\left\{A^{(1,3)}\right\} \subseteq\left\{A^{-}\right\}, \quad\left\{A^{(1,2,4)}\right\} \subseteq\left\{A^{(1,4)}\right\} \subseteq\left\{A^{-}\right\}
\end{aligned}
$$

One of the main studies in the theory of generalized inverses is to find generalized inverses of block matrices and their properties. For the simplest block matrix $M=[A, B]$, where $A \in \mathbb{C}^{m \times n}$ and $B \in \mathbb{C}^{m \times k}$, there have been many results on its generalized inverses. For example, the Moore-Penrose inverse of $[A, B]^{\dagger}$ can be represented as

$$
[A, B]^{\dagger}=\left[\begin{array}{c}
A^{*}\left(A A^{*}+B B^{*}\right)^{\dagger} \\
B^{*}\left(A A^{*}+B B^{*}\right)^{\dagger}
\end{array}\right]
$$

which is derived from the equality $M^{\dagger}=M^{*}\left(M M^{*}\right)^{\dagger}$. Another representation of $[A, B]^{\dagger}$ in Mihályffy $[4]$ is

$$
[A, B]^{\dagger}=\left[\begin{array}{c}
\left(I_{n}+T T^{*}\right)^{-1}\left(A^{\dagger}-A^{\dagger} B S^{\dagger}\right) \\
T^{*}\left(I_{n}+T T^{*}\right)^{-1}\left(A^{\dagger}-A^{\dagger} B S^{\dagger}\right)+S^{\dagger}
\end{array}\right],
$$

where $S=E_{A} B$ and $T=A^{\dagger} B F_{S}$. Moreover, it can easily be verified by definition that

$$
\begin{gathered}
\left\{\left[\begin{array}{c}
\left(A-B B^{-} A\right)^{-}\left(I_{m}-B B^{-}\right) \\
B^{-}-B^{-} A\left(A-B B^{-} A\right)^{-}\left(I_{m}-B B^{-}\right)
\end{array}\right]\right\} \subseteq\left\{[A, B]^{-}\right\}, \\
\left\{\left[\begin{array}{c}
A^{-}-A^{-} B\left(B-A A^{-} B\right)^{-}\left(I_{m}-A A^{-}\right) \\
\left(B-A A^{-} B\right)^{-}\left(I_{m}-A A^{-}\right)
\end{array}\right]\right\} \subseteq\left\{[A, B]^{-}\right\}, \\
{\left[\begin{array}{c}
\left(E_{B} A\right)^{\dagger} \\
B^{\dagger}-B^{\dagger} A\left(E_{B} A\right)^{\dagger}
\end{array}\right] \in\left\{[A, B]^{(1,2,4)}\right\}, \quad\left[\begin{array}{c}
A^{\dagger}-A^{\dagger} B\left(E_{A} B\right)^{\dagger} \\
\left(E_{A} B\right)^{\dagger}
\end{array}\right] \in\left\{[A, B]^{(1,2,4)}\right\}}
\end{gathered}
$$

hold true. 
It has been shown that the rank of matrix provides a powerful tool for characterizing various equalities for generalized inverses of matrices. For example, Tian [9] established the following three equalities:

$$
\begin{aligned}
r\left([A, B]-[A, B]\left[\begin{array}{l}
A^{\dagger} \\
B^{\dagger}
\end{array}\right][A, B]\right) & =r[A, B]+2 r\left(A^{*} B\right)-r(A)-r(B), \\
r\left([A, B]^{\dagger}-\left[\begin{array}{c}
A^{\dagger} \\
B^{\dagger}
\end{array}\right]\right) & =r\left[A A^{*} B, B B^{*} A\right], \\
r\left([A, B]^{\dagger}-\left[\begin{array}{c}
\left(E_{B} A\right)^{\dagger} \\
\left(E_{A} B\right)^{\dagger}
\end{array}\right]\right) & =r(A)+r(B)-r[A, B] .
\end{aligned}
$$

Let the right-hand side of (1.9) be zero, we see that $\left[\begin{array}{c}A^{\dagger} \\ B^{\dagger}\end{array}\right] \in\left\{[A, B]^{-}\right\}$if and only if $r[A, B]=r(A)+r(B)-2 r\left(A^{*} B\right)$. However, the inequality $r[A, B] \geq r(A)+$ $r(B)-r\left(A^{*} B\right)$ always holds. Hence $r[A, B]=r(A)+r(B)-2 r\left(A^{*} B\right)$ is equivalent to $A^{*} B=0$. Let the right-hand side of $(1.10)$ be zero, we see that $[A, B]^{\dagger}=\left[\begin{array}{l}A^{\dagger} \\ B^{\dagger}\end{array}\right]$ if and only if $A^{*} B=0$. Let the right-hand side of (1.11) be zero, we see that $[A, B]^{\dagger}=\left[\begin{array}{c}\left(E_{B} A\right)^{\dagger} \\ \left(E_{A} B\right)^{\dagger}\end{array}\right]$ if and only if $r[A, B]=r(A)+r(B)$. Motivated by these results, we shall establish a variety of new rank formulas for the difference

$$
[A, B]^{(i, \ldots, j)}-\left[\begin{array}{l}
A^{(i, \ldots, j)} \\
B^{(i, \ldots, j)}
\end{array}\right],
$$

and then use the formulas to characterize the corresponding matrix equalities for generalized inverses.

Some useful rank formulas for partitioned matrices due to Marsaglia and Styan [3] are given in the following lemma.

Lemma 1.1. Let $A \in \mathbb{C}^{m \times n}, B \in \mathbb{C}^{m \times k}, C \in \mathbb{C}^{l \times n}$ and $D \in \mathbb{C}^{l \times k}$. Then

$$
\begin{aligned}
r[A, B] & =r(A)+r\left[\left(I_{m}-A A^{-}\right) B\right]=r(B)+r\left[\left(I_{m}-B B^{-}\right) A\right], \\
r\left[\begin{array}{l}
A \\
C
\end{array}\right] & =r(A)+r\left[C\left(I_{n}-A^{-} A\right)\right]=r(C)+r\left[A\left(I_{n}-C^{-} C\right)\right], \\
r\left[\begin{array}{cc}
A & B \\
C & 0
\end{array}\right] & =r(B)+r(C)+r\left[\left(I_{m}-B B^{-}\right) A\left(I_{n}-C^{-} C\right)\right] .
\end{aligned}
$$

If $\mathscr{R}(B) \subseteq \mathscr{R}(A)$ and $\mathscr{R}\left(C^{*}\right) \subseteq \mathscr{R}\left(A^{*}\right)$, then

$$
r\left[\begin{array}{cc}
A & B \\
C & D
\end{array}\right]=r(A)+r\left(D-C A^{\dagger} B\right) .
$$

Applying (1.15) and $A^{*}\left(A^{*} A A^{*}\right)^{\dagger} A^{*}=A^{\dagger}$ (see Zlobec [12]) to a general Schur complement $D-C A^{\dagger} B$ gives the following rank formula:

$$
r\left(D-C A^{\dagger} B\right)=r\left[\begin{array}{cc}
A^{*} A A^{*} & A^{*} B \\
C A^{*} & D
\end{array}\right]-r(A) .
$$


This formula can be used to find the rank of any matrix expression involving the Moore-Penrose inverse. In fact, the equalities in (1.9), (1.10) and (1.11) are derived from (1.16).

Because generalized inverse of a matrix is not necessarily unique, the rank of a matrix expression involving generalized inverses of matrices may vary with respect to the choice of the generalized inverses. Suppose $f\left(A_{1}^{-}, \ldots, A_{p}^{-}\right)$and $g\left(B_{1}^{-}, \ldots, B_{q}^{-}\right)$ are two expressions consisting of generalized inverses of matrices. Then there are $A_{1}^{-}, \ldots, A_{p}^{-}, B_{1}^{-}, \ldots, B_{q}^{-}$such that $f\left(A_{1}^{-}, \ldots, A_{p}^{-}\right)=g\left(B_{1}^{-}, \ldots, B_{q}^{-}\right)$if and only if

$$
\min _{A_{1}^{-}, \ldots, A_{p}^{-}, B_{1}^{-}, \ldots, B_{q}^{-}} r\left[f\left(A_{1}^{-}, \ldots, A_{p}^{-}\right)-g\left(B_{1}^{-}, \ldots, B_{q}^{-}\right)\right]=0 .
$$

One of the simplest matrix expressions involving a generalized inverse is the Schur complement $D-C A^{-} B$. In Tian [10], a group of formulas for the extremal ranks of the Schur complement $D-C A^{(i, \ldots, j)} B$ with respect to $A^{(i, \ldots, j)}$ are derived. The following lemma presents some special cases of these formulas.

Lemma 1.2. Let $A \in \mathbb{C}^{m \times n}$ and $G \in \mathbb{C}^{n \times m}$. Then

(1.18) $\min _{A^{(1,2)}} r\left(A^{(1,2)}-G\right)=\max \{r(A-A G A), r(G)+r(A)-r(G A)-r(A G)\}$,

(1.19) $\min _{A^{(1,3)}} r\left(A^{(1,3)}-G\right)=r\left(A^{*} A G-A^{*}\right)$

(1.20) $\min _{A^{(1,4)}} r\left(A^{(1,4)}-G\right)=r\left(G A A^{*}-A^{*}\right)$,

(1.21) $\min _{A^{(1,2,3)}} r\left(A^{(1,2,3)}-G\right)=r\left(A^{*} A G-A^{*}\right)+r\left[\begin{array}{c}A^{*} \\ G\end{array}\right]-r\left[\begin{array}{c}A^{*} \\ A G\end{array}\right]$,

(1.22) $\min _{A^{(1,2,4)}} r\left(A^{(1,2,4)}-G\right)=r\left(G A A^{*}-A^{*}\right)+r\left[A^{*}, G\right]-r\left[A^{*}, G A\right]$,

(1.23) $\min _{A^{(1,3,4)}} r\left(A^{(1,3,4)}-G\right)=r\left(A^{*} A G-A^{*}\right)+r\left(G A A^{*}-A^{*}\right)-r(A-A G A)$,

$$
r\left(A^{\dagger}-G\right)=r\left[\begin{array}{cc}
A^{*} A A^{*} & A^{*} \\
A^{*} & G
\end{array}\right]-r(A) .
$$

Let the right-hand sides of (1.17)-(1.24) be zero, we can obtain necessary and sufficient conditions for $G \in\left\{A^{-}\right\},\left\{A^{(1,2)}\right\},\left\{A^{(1,3)}\right\},\left\{A^{(1,4)}\right\},\left\{A^{(1,2,3)}\right\},\left\{A^{(1,2,4)}\right\}$, $\left\{A^{(1,3,4)}\right\}$ and $G=A^{\dagger}$ to hold. If $A$ and $G$ in (1.17)-(1.24) are taken as matrix products, matrix sums and partitioned matrices, then many valuable results can be derived from the corresponding rank formulas.

Some other rank formulas used in the context are given below:

$$
\min _{X} r(A-B X C)=r[A, B]+r\left[\begin{array}{l}
A \\
C
\end{array}\right]-r\left[\begin{array}{ll}
A & B \\
C & 0
\end{array}\right],
$$

where $A \in \mathbb{C}^{m \times n}, B \in \mathbb{C}^{m \times k}$ and $C \in \mathbb{C}^{l \times n}$; see Tian [8]. In particular,

$$
\min _{X} r(A-B X)=r[A, B]-r(A)=r\left(B-A A^{\dagger} B\right) .
$$


The formula for minimal rank of $A-B_{1} X_{1} C_{1}-B_{2} X_{2} C_{2}$ is (1.27) $\min _{X_{1}, X_{2}} r\left(A-B_{1} X_{1} C_{1}-B_{2} X_{2} C_{2}\right)=r\left[\begin{array}{c}A \\ C_{1} \\ C_{2}\end{array}\right]+r\left[A, B_{1}, B_{2}\right]+\max \left\{r_{1}, r_{2}\right\}$, where

$$
\begin{aligned}
& r_{1}=r\left[\begin{array}{cc}
A & B_{1} \\
C_{2} & 0
\end{array}\right]-r\left[\begin{array}{ccc}
A & B_{1} & B_{2} \\
C_{2} & 0 & 0
\end{array}\right]-r\left[\begin{array}{cc}
A & B_{1} \\
C_{1} & 0 \\
C_{2} & 0
\end{array}\right], \\
& r_{2}=r\left[\begin{array}{cc}
A & B_{2} \\
C_{1} & 0
\end{array}\right]-r\left[\begin{array}{ccc}
A & B_{1} & B_{2} \\
C_{1} & 0 & 0
\end{array}\right]-r\left[\begin{array}{cc}
A & B_{2} \\
C_{1} & 0 \\
C_{2} & 0
\end{array}\right] ;
\end{aligned}
$$

see Tian [6]. The following is also given [6]:

$$
\min _{A^{-}} r\left(C A^{-} B\right)=r\left[\begin{array}{cc}
A & B \\
C & 0
\end{array}\right]-r\left[\begin{array}{l}
A \\
C
\end{array}\right]-r[A, B]+r(A) .
$$

2. Main results. We first establish a set of rank formulas for the difference $[A, B]^{(i, \ldots, j)}-\left[\begin{array}{c}A^{\dagger} \\ B^{\dagger}\end{array}\right]$.

Then

Theorem 2.1. Let $A \in \mathbb{C}^{m \times n}, B \in \mathbb{C}^{m \times k}$ and let $M=[A, B], G=\left[\begin{array}{l}A^{\dagger} \\ B^{\dagger}\end{array}\right]$.

$$
\begin{aligned}
\min _{M^{-}} r\left(M^{-}-G\right) & =r(M)+2 r\left(A^{*} B\right)-r(A)-r(B), \\
\min _{M^{(1,2)}} r\left(M^{(1,2)}-G\right) & =r(M)+2 r\left(A^{*} B\right)-r(A)-r(B), \\
\min _{M^{(1,3)}} r\left(M^{(1,3)}-G\right) & =r(M)+2 r\left(A^{*} B\right)-r(A)-r(B), \\
\min _{M^{(1,4)}} r\left(M^{(1,4)}-G\right) & =r\left[A A^{*} B, B B^{*} A\right], \\
\min _{M^{(1,2,3)}} r\left(M^{(1,2,3)}-G\right) & =r(M)+2 r\left(A^{*} B\right)-r(A)-r(B), \\
\min _{M^{(1,2,4)}} r\left(M^{(1,2,4)}-G\right) & =r\left[A A^{*} B, B B^{*} A\right], \\
\min _{M^{(1,3,4)}} r\left(M^{(1,3,4)}-G\right) & =r\left[A A^{*} B, B B^{*} A\right], \\
r\left(M^{\dagger}-G\right) & =r\left[A A^{*} B, B B^{*} A\right] .
\end{aligned}
$$

Hence

$$
G \in\left\{M^{-}\right\} \Leftrightarrow M^{\dagger}=G \Leftrightarrow A^{*} B=0 .
$$


Proof. Applying (1.17) to the difference $M^{-}-G$ gives

$$
\min _{M^{-}} r\left(M^{-}-G\right)=r(M-M G M),
$$

where

$$
M-M G M=M-\left(A A^{\dagger}+B B^{\dagger}\right) M=-\left[B B^{\dagger} A, A A^{\dagger} B\right] .
$$

It is shown in [9] that

$$
r\left[B B^{\dagger} A, A A^{\dagger} B\right]=r[A, B]+2 r\left(A^{*} B\right)-r(A)-r(B) .
$$

Substituting (2.11) into (2.10) yields (2.1). Applying (1.18) to $M^{(1,2)}-G$ gives

$$
\min _{M^{(1,2)}} r\left(M^{(1,2)}-G\right)=\max \{r(M-M G M), r(G)+r(M)-r(G M)-r(M G)\} .
$$

It is easy to verify that

$$
\begin{gathered}
r(G)=r\left[\begin{array}{l}
A^{*} \\
B^{*}
\end{array}\right]=r(M), r(G M)=r\left(\left[\begin{array}{l}
A^{*} \\
B^{*}
\end{array}\right][A, B]\right)=r(M), \\
r(M G)=r\left(A A^{\dagger}+B B^{\dagger}\right)=r\left[A A^{\dagger}, B B^{\dagger}\right]=r(M) .
\end{gathered}
$$

Hence,

$$
\min _{M^{(1,2)}} r\left(M^{(1,2)}-G\right)=r(M-M G M)=\min _{M^{-}} r\left(M^{-}-G\right) .
$$

Applying (1.19) to $M^{(1,3)}-G$ gives

$$
\min _{M^{(1,3)}} r\left(M^{(1,3)}-G\right)=r\left(M^{*} M G-M^{*}\right),
$$

where

$$
M^{*} M G-M^{*}=\left[\begin{array}{l}
A^{*}+A^{*} B B^{\dagger} \\
B^{*}+B^{*} A A^{\dagger}
\end{array}\right]-\left[\begin{array}{c}
A^{*} \\
B^{*}
\end{array}\right]=\left[\begin{array}{c}
A^{*} B B^{\dagger} \\
B^{*} A A^{\dagger}
\end{array}\right]=\left[B B^{\dagger} A, A A^{\dagger} B\right]^{*} .
$$

Hence, (2.3) follows from (2.11). From (1.20)

$$
\min _{M^{(1,4)}} r\left(M^{(1,4)}-G\right)=r\left(G M M^{*}-M^{*}\right),
$$

where $G M M^{*}-M^{*}=\left[\begin{array}{c}A^{\dagger} B B^{*} \\ B^{\dagger} A A^{*}\end{array}\right]$. Hence

$$
r\left(G M M^{*}-M^{*}\right)=r\left[\begin{array}{l}
A^{\dagger} B B^{*} \\
B^{\dagger} A A^{*}
\end{array}\right]=r\left[\begin{array}{l}
A^{*} B B^{*} \\
B^{*} A A^{*}
\end{array}\right]=r\left[A A^{*} B, B B^{*} A\right],
$$

establishing (2.4). Equalities (2.5)-(2.7) can be shown by (1.21)-(1.23), and details are omitted. Eq.(2.8) is (1.10). 
A set of rank formulas for the difference $[A, B]^{\dagger}-\left[\begin{array}{l}A^{(i, \ldots, j)} \\ B^{(i, \ldots, j)}\end{array}\right]$ are given below.

Theorem 2.2. Let $A \in \mathbb{C}^{m \times n}$ and $B \in \mathbb{C}^{m \times k}$, and let $M=[A, B]$. Then

$$
\begin{aligned}
\min _{A^{-}, B^{-}} r\left(M^{\dagger}-\left[\begin{array}{l}
A^{-} \\
B^{-}
\end{array}\right]\right) & =2 r(A)+2 r(B)-2 r(M), \\
\min _{A^{(1,2)}, B^{(1,2)}} r\left(M^{\dagger}-\left[\begin{array}{l}
A^{(1,2)} \\
B^{(1,2)}
\end{array}\right]\right) & =2 r(A)+2 r(B)-2 r(M), \\
\min _{A^{(1,3)}, B^{(1,3)}} r\left(M^{\dagger}-\left[\begin{array}{l}
A^{(1,3)} \\
B^{(1,3)}
\end{array}\right]\right) & =r\left[B B^{*} A, A A^{*} B\right], \\
\min _{A^{(1,4)}, B^{(1,4)}} r\left(M^{\dagger}-\left[\begin{array}{l}
A^{(1,4)} \\
B^{(1,4)}
\end{array}\right]\right) & =2 r(A)+2 r(B)-2 r(M), \\
\min _{A^{(1,2,3)}, B^{(1,2,3)}} r\left(M^{\dagger}-\left[\begin{array}{l}
A^{(1,2,3)} \\
B^{(1,2,3)}
\end{array}\right]\right) & =r\left[B B^{*} A, A A^{*} B\right], \\
\min _{A^{(1,2,4)}, B^{(1,2,4)}} r\left(M^{\dagger}-\left[\begin{array}{l}
A^{(1,2,4)} \\
B^{(1,2,4)}
\end{array}\right]\right) & =2 r(A)+2 r(B)-2 r(M), \\
\min _{A^{(1,3,4)}, B^{(1,3,4)}} r\left(M^{\dagger}-\left[\begin{array}{l}
A^{(1,3,4)} \\
B^{(1,3,4)}
\end{array}\right]\right) & =r\left[B B^{*} A, A A^{*} B\right] .
\end{aligned}
$$

Hence,

(a) There are $A^{-}\left(A^{(1,2)}, A^{(1,2,4)}\right)$ and $B^{-}\left(B^{(1,2)}, B^{(1,2,4)}\right)$ such that

$$
M^{\dagger}=\left[\begin{array}{l}
A^{-} \\
B^{-}
\end{array}\right]\left(M^{\dagger}=\left[\begin{array}{l}
A^{(1,2)} \\
B^{(1,2)}
\end{array}\right], M^{\dagger}=\left[\begin{array}{l}
A^{(1,2,4)} \\
B^{(1,2,4)}
\end{array}\right]\right)
$$

if and only if $r(M)=r(A)+r(B)$, i.e., $\mathscr{R}(A) \cap \mathscr{R}(B)=\{0\}$.

(b) There are $A^{(1,3)}\left(A^{(1,2,3)}, A^{(1,3,4)}\right)$ and $B^{(1,3)}\left(B^{(1,2,3)}, B^{(1,3,4)}\right)$ such that

$$
M^{\dagger}=\left[\begin{array}{l}
A^{(1,3)} \\
B^{(1,3)}
\end{array}\right]\left(M^{\dagger}=\left[\begin{array}{l}
A^{(1,2,3)} \\
B^{(1,2,3)}
\end{array}\right], M^{\dagger}=\left[\begin{array}{l}
A^{(1,3,4)} \\
B^{(1,3,4)}
\end{array}\right]\right)
$$

if and only if $A^{*} B=0$.

Proof. From (1.1)

$$
\begin{aligned}
M^{\dagger}-\left[\begin{array}{l}
A^{-} \\
B^{-}
\end{array}\right] & =M^{\dagger}-\left[\begin{array}{l}
A^{\dagger}+F_{A} V_{1}+W_{1} E_{A} \\
B^{\dagger}+F_{B} V_{2}+W_{2} E_{B}
\end{array}\right] \\
& =M^{\dagger}-\left[\begin{array}{c}
A^{\dagger} \\
B^{\dagger}
\end{array}\right]-\left[\begin{array}{cc}
F_{A} & 0 \\
0 & F_{B}
\end{array}\right]\left[\begin{array}{l}
V_{1} \\
V_{2}
\end{array}\right]-\left[\begin{array}{l}
W_{1} E_{A} \\
W_{2} E_{B}
\end{array}\right],
\end{aligned}
$$

where $V_{1}, W_{1}, V_{2}$ and $W_{2}$ are arbitrary. From (1.26)

$$
\begin{aligned}
& \min _{V_{1}, V_{2}} r\left(M^{\dagger}-\left[\begin{array}{c}
A^{\dagger} \\
B^{\dagger}
\end{array}\right]-\left[\begin{array}{cc}
F_{A} & 0 \\
0 & F_{B}
\end{array}\right]\left[\begin{array}{l}
V_{1} \\
V_{2}
\end{array}\right]-\left[\begin{array}{l}
W_{1} E_{A} \\
W_{2} E_{B}
\end{array}\right]\right) \\
& =r\left\{\left[\begin{array}{cc}
A^{\dagger} A & 0 \\
0 & B^{\dagger} B
\end{array}\right]\left(M^{\dagger}-\left[\begin{array}{c}
A^{\dagger} \\
B^{\dagger}
\end{array}\right]-\left[\begin{array}{l}
W_{1} E_{A} \\
W_{2} E_{B}
\end{array}\right]\right)\right\}
\end{aligned}
$$




$$
\begin{aligned}
& =r\left(M^{\dagger}-\left[\begin{array}{c}
A^{\dagger} \\
B^{\dagger}
\end{array}\right]-\left[\begin{array}{c}
A^{\dagger} A W_{1} E_{A} \\
B^{\dagger} B W_{2} E_{B}
\end{array}\right]\right) \\
& =r\left(M^{\dagger}-\left[\begin{array}{c}
A^{\dagger} \\
B^{\dagger}
\end{array}\right]-\left[\begin{array}{c}
A^{\dagger} A \\
0
\end{array}\right] W_{1} E_{A}-\left[\begin{array}{c}
0 \\
B^{\dagger} B
\end{array}\right] W_{2} E_{B}\right) .
\end{aligned}
$$

Let $N=\left[\begin{array}{c}A^{\dagger} \\ B^{\dagger}\end{array}\right], P_{1}=\left[\begin{array}{c}A^{\dagger} A \\ 0\end{array}\right]$ and $P_{2}=\left[\begin{array}{c}0 \\ B^{\dagger} B\end{array}\right]$. Then applying (1.27) gives

$$
\begin{aligned}
& \min _{W_{1}, W_{2}} r\left(M^{\dagger}-N-P_{1} W_{1} E_{A}-P_{2} W_{2} E_{B}\right) \\
& =r\left[M^{\dagger}-N, P_{1}, P_{2}\right]+r\left[\begin{array}{c}
M^{\dagger}-N \\
E_{A} \\
E_{B}
\end{array}\right]+\max \left\{r_{1}, r_{2}\right\},
\end{aligned}
$$

where

$$
\begin{aligned}
& r_{1}=r\left[\begin{array}{cc}
M^{\dagger}-N & P_{1} \\
E_{B} & 0
\end{array}\right]-r\left[\begin{array}{ccc}
M^{\dagger}-N & P_{1} & P_{2} \\
E_{B} & 0 & 0
\end{array}\right]-r\left[\begin{array}{cc}
M^{\dagger}-N & P_{1} \\
E_{A} & 0 \\
E_{B} & 0
\end{array}\right], \\
& r_{2}=r\left[\begin{array}{cc}
M^{\dagger}-N & P_{2} \\
E_{A} & 0
\end{array}\right]-r\left[\begin{array}{ccc}
M^{\dagger}-N & P_{1} & P_{2} \\
E_{A} & 0 & 0
\end{array}\right]-r\left[\begin{array}{cc}
M^{\dagger}-N & P_{2} \\
E_{A} & 0 \\
E_{B} & 0
\end{array}\right] .
\end{aligned}
$$

Substituting (1.8) into the eight partitioned matrices in (2.21) and simplifying by (1.12), (1.13) and (1.14) give us the following results:

$$
\begin{aligned}
& r\left[M^{\dagger}-N, P_{1}, P_{2}\right]=r(A)+r(B), \quad r\left[\begin{array}{c}
M^{\dagger}-N \\
E_{A} \\
E_{B}
\end{array}\right]=m+r(A)+r(B)-r(M), \\
& r\left[\begin{array}{cc}
M^{\dagger}-N & P_{1} \\
E_{B} & 0
\end{array}\right]=m+2 r(A)-r(M), \quad r\left[\begin{array}{ccc}
M^{\dagger}-N & P_{1} & P_{2} \\
E_{B} & 0 & 0
\end{array}\right]=m+r(A), \\
& r\left[\begin{array}{cc}
M^{\dagger}-N & P_{1} \\
E_{A} & 0 \\
E_{B} & 0
\end{array}\right]=m+r(A), \quad r\left[\begin{array}{cc}
M^{\dagger}-N & P_{2} \\
E_{A} & 0
\end{array}\right]=m+2 r(B)-r(M), \\
& r\left[\begin{array}{ccc}
M^{\dagger}-N & P_{1} & P_{2} \\
E_{A} & 0 & 0
\end{array}\right]=m+r(B), \quad r\left[\begin{array}{cc}
M^{\dagger}-N & P_{2} \\
E_{A} & 0 \\
E_{A} & 0
\end{array}\right]=m+r(B) .
\end{aligned}
$$

The details are quite tedious, and therefore are omitted here. Substituting these eight results into (2.21) and then (2.21) into (2.20) yields (2.12). From (1.3)

(2.22) $M^{\dagger}-\left[\begin{array}{l}A^{(1,3)} \\ B^{(1,3)}\end{array}\right]=M^{\dagger}-\left[\begin{array}{l}A^{\dagger}+F_{A} V_{1} \\ B^{\dagger}+F_{B} V_{2}\end{array}\right]=M^{\dagger}-\left[\begin{array}{c}A^{\dagger} \\ B^{\dagger}\end{array}\right]-\left[\begin{array}{cc}F_{A} & 0 \\ 0 & F_{B}\end{array}\right]\left[\begin{array}{l}V_{1} \\ V_{2}\end{array}\right]$,

where $V_{1}$ and $V_{2}$ are arbitrary. Applying (1.26) to this expression gives

$$
\min _{V_{1}, V_{2}} r\left(M^{\dagger}-N-\left[\begin{array}{cc}
F_{A} & 0 \\
0 & F_{B}
\end{array}\right]\left[\begin{array}{l}
V_{1} \\
V_{2}
\end{array}\right]\right)=r\left(\left[\begin{array}{cc}
A^{\dagger} A & 0 \\
0 & B^{\dagger} B
\end{array}\right]\left(M^{\dagger}-N\right)\right)
$$




$$
\begin{aligned}
& =r\left(M^{\dagger}-N\right) \\
& =r\left[B B^{*} A, A A^{*} B\right] \quad(\text { by }(1.10)),
\end{aligned}
$$

as required for (2.14). From (1.4)

$$
\begin{aligned}
M^{\dagger}-\left[\begin{array}{c}
A^{(1,4)} \\
B^{(1,4)}
\end{array}\right] & =M^{\dagger}-\left[\begin{array}{c}
A^{\dagger}+W_{1} E_{A} \\
B^{\dagger}+W_{2} E_{B}
\end{array}\right] \\
& =M^{\dagger}-N-\left[\begin{array}{c}
I_{n} \\
0
\end{array}\right] W_{1} E_{A}-\left[\begin{array}{c}
0 \\
I_{k}
\end{array}\right] W_{2} E_{B},
\end{aligned}
$$

where $W_{1}$ and $W_{2}$ are arbitrary. Applying (1.26) to (2.23) and simplifying by (1.12), (1.13) and (1.14) yield (2.15). The details are omitted here. Observe that

$$
\min _{A^{(1,3)}} r\left(M^{\dagger}-\left[\begin{array}{l}
A^{(1,3)} \\
B^{(1,3)}
\end{array}\right]\right) \leqslant \min _{A^{(1,2,3)}} r\left(M^{\dagger}-\left[\begin{array}{l}
A^{(1,2,3)} \\
B^{(1,2,3)}
\end{array}\right]\right) \leqslant r\left(M^{\dagger}-N\right)
$$

and

$$
\min _{B^{(1,3)}} r\left(M^{\dagger}-\left[\begin{array}{c}
A^{(1,3)} \\
B^{(1,3)}
\end{array}\right]\right) \leqslant \min _{A^{(1,3,4)}} r\left(M^{\dagger}-\left[\begin{array}{c}
A^{(1,3,4)} \\
B^{(1,3,4)}
\end{array}\right]\right) \leqslant r\left(M^{\dagger}-N\right) .
$$

Applying (1.10) and (2.14) to these two inequalities gives (2.16) and (2.18). From (1.6)

$$
\begin{aligned}
M^{\dagger}-\left[\begin{array}{c}
A^{(1,2,4)} \\
B^{(1,2,4)}
\end{array}\right] & =M^{\dagger}-\left[\begin{array}{c}
A^{\dagger}+A^{\dagger} A W_{1} E_{A} \\
B^{\dagger}+B^{\dagger} B W_{2} E_{B}
\end{array}\right] \\
& =M^{\dagger}-N-\left[\begin{array}{c}
A^{\dagger} A \\
0
\end{array}\right] W_{1} E_{A}-\left[\begin{array}{c}
0 \\
B^{\dagger} B
\end{array}\right] W_{2} E_{B}
\end{aligned}
$$

where $W_{1}$ and $W_{2}$ are arbitrary. Applying (1.27) to (2.24) and simplifying by (1.12), (1.13) and (1.14) yield (2.17). Also note that

$$
\min _{A^{-}, B^{-}} r\left(M^{\dagger}-\left[\begin{array}{c}
A^{-} \\
B^{-}
\end{array}\right]\right) \leqslant \min _{A_{B^{(1,2)}}^{(1,2)}} r\left(M^{\dagger}-\left[\begin{array}{c}
A^{(1,2)} \\
B^{(1,2)}
\end{array}\right]\right) \leqslant \min _{A_{B^{(1,2,2)}}^{(1,2)}} r\left(M^{\dagger}-\left[\begin{array}{l}
A^{(1,2,4)} \\
B^{(1,2,4)}
\end{array}\right]\right) .
$$

Applying (2.12) and (2.17) to this gives (2.13).

Some rank formulas for $[A, B]^{(i, \ldots, j)}-\left[\begin{array}{l}A^{(i, \ldots, j)} \\ B^{(i, \ldots, j)}\end{array}\right]$ are given below.

Theorem 2.3. Let $A \in \mathbb{C}^{m \times n}$ and $B \in \mathbb{C}^{m \times k}$, and let $M=[A, B]$. Then

$$
\min _{M^{-}, A^{-}, B^{-}} r\left(M^{-}-\left[\begin{array}{l}
A^{-} \\
B^{-}
\end{array}\right]\right)=r(A)+r(B)-r(M)
$$




$$
\begin{aligned}
& (2.26) \min _{M^{(1,3)}, A^{(1,3)}, B^{(1,3)}} r\left(M^{(1,3)}-\left[\begin{array}{l}
A^{(1,3)} \\
B^{(1,3)}
\end{array}\right]\right)=r(M)+2 r\left(A^{*} B\right)-r(A)-r(B), \\
& (2.27) \min _{M^{(1,4)}, A^{(1,4)}, B^{(1,4)}} r\left(M^{(1,4)}-\left[\begin{array}{l}
A^{(1,4)} \\
B^{(1,4)}
\end{array}\right]\right)=r(A)+r(B)-r(M), \\
& (2.28) \min _{M^{(1,2,3)}} r\left(M^{(1,2,3)}-\left[\begin{array}{l}
A^{(1,2,3)} \\
B^{(1,2,3)}
\end{array}\right]\right)=r(M)+2 r\left(A^{*} B\right)-r(A)-r(B) . \\
& A^{(1,2,3)}, B^{(1,2,3)}
\end{aligned}
$$

Hence,

(a) The following statements are equivalent:
(i) There are $A^{-}$and $B^{-}$such that $\left[\begin{array}{l}A^{-} \\ B^{-}\end{array}\right] \in\left\{M^{-}\right\}$.
(ii) There are $A^{(1,4)}$ and $B^{(1,4)}$ such that $\left[\begin{array}{l}A^{(1,4)} \\ B^{(1,4)}\end{array}\right] \in\left\{M^{(1,4)}\right\}$.

(iii) $\mathscr{R}(A) \cap \mathscr{R}(B)=\{0\}$.

(b) The following statements are equivalent:

(i) There are $A^{(1,3)}$ and $B^{(1,3)}$ such that $\left[\begin{array}{l}A^{(1,3)} \\ B^{(1,3)}\end{array}\right] \in\left\{M^{(1,3)}\right\}$.

(ii) There are $A^{(1,2,3)}$ and $B^{(1,2,3)}$ such that $\left[\begin{array}{l}A^{(1,2,3)} \\ B^{(1,2,3)}\end{array}\right] \in\left\{M^{(1,2,3)}\right\}$.

(iii) $M^{\dagger}=\left[\begin{array}{l}A^{\dagger} \\ B^{\dagger}\end{array}\right]$.

(iv) $A^{*} B=0$.

Proof. From (1.17)

$$
\min _{M^{-}} r\left(M^{-}-\left[\begin{array}{l}
A^{-} \\
B^{-}
\end{array}\right]\right)=r\left(M-M\left[\begin{array}{l}
A^{-} \\
B^{-}
\end{array}\right] M\right) .
$$

Expanding the difference on the right-hand side of (2.29) yields

$$
M-M\left[\begin{array}{l}
A^{-} \\
B^{-}
\end{array}\right] M=\left[B B^{-} A, A A^{-} B\right]=\left[B B^{\dagger} A, A A^{\dagger} B\right]+\left[B W_{1} E_{B} A, A W_{2} E_{A} B\right],
$$

where $W_{1}$ and $W_{2}$ are two arbitrary matrices. Applying (1.27) to the right-hand side and simplifying by $(1.12),(1.13)$ and $(1.14)$ give us

$$
\begin{aligned}
& \min _{A^{-}, B^{-}} r\left(M-M\left[\begin{array}{l}
A^{-} \\
B^{-}
\end{array}\right] M\right) \\
& =\min _{V_{1}, V_{2}} r\left(\left[B B^{\dagger} A, A A^{\dagger} B\right]+\left[B V_{1} E_{B} A, A W_{2} E_{A} B\right]\right) \\
& =r(A)+r(B)-r(M) .
\end{aligned}
$$

Combining (2.29) and (2.30) results in (2.25). Eqs.(2.26), (2.27) and (2.28) can be shown by (1.19), (1.20), (1.21) and (1.27). The details are omitted here. 
To find

$$
\begin{gathered}
\min _{M^{(1,2)}} r\left(M^{(1,2)}-\left[\begin{array}{l}
A^{(1,2)} \\
B^{(1,2)}
\end{array}\right]\right), \\
A^{(1,2)}, B^{(1,2)} \\
\min _{M^{(1,3,4)}} r\left(M^{(1,2,4)}, B^{(1,2,4)}\right. \\
A^{(1,3,4)}, B^{(1,3,4)}
\end{gathered}
$$

a formula for the minimal rank of $A+B_{1} X_{1} C_{1}+B_{2} X_{2} C_{2}+B_{3} X_{3} C_{3}$ with respect to $X_{1}, X_{2}$ and $X_{3}$ is needed. However, such a formula has not been established at present; see [7].

In addition to $\left[\begin{array}{c}A^{-} \\ B^{-}\end{array}\right],[A, B]$ may have generalized inverses like $\left[\begin{array}{c}A^{-} \\ X\end{array}\right]$ or $\left[\begin{array}{c}Y \\ B^{-}\end{array}\right]$ for some matrices $X$ and $Y$.

TheOREm 2.4. Let $A \in \mathbb{C}^{m \times n}, B \in \mathbb{C}^{m \times k}, X \in \mathbb{C}^{k \times m}, Y \in \mathbb{C}^{n \times m}$, and let $M=[A, B]$. Then

$$
\begin{aligned}
\min _{X} r\left(M-M\left[\begin{array}{c}
A^{\dagger} \\
X
\end{array}\right] M\right) & =\min _{Y} r\left(M-M\left[\begin{array}{c}
Y \\
B^{\dagger}
\end{array}\right] M\right) \\
& =r[A, B]+r\left(A^{*} B\right)-r(A)-r(B) .
\end{aligned}
$$

Hence the following statement are equivalent:

(a) There is $X$ such that $\left[\begin{array}{c}A^{\dagger} \\ X\end{array}\right] \in\left\{M^{-}\right\}$.

(b) There is $Y$ such that $\left[\begin{array}{c}Y \\ B^{\dagger}\end{array}\right] \in\left\{M^{-}\right\}$.

(c) $r[A, B]=r(A)+r(B)-r\left(A^{*} B\right)$.

Proof. Applying (1.25) and simplifying by elementary block matrix operations give us

$$
\begin{aligned}
& \min _{X} r\left(M-M\left[\begin{array}{c}
A^{\dagger} \\
X
\end{array}\right] M\right) \\
& =\min _{X} r\left(\left[0, B-A A^{\dagger} B\right]-B X[A, B]\right) \\
& =r\left[B-A A^{\dagger} B, B\right]+r\left[\begin{array}{cc}
0 & B-A A^{\dagger} B \\
A & B
\end{array}\right]-r\left[\begin{array}{ccc}
0 & B-A A^{\dagger} B & B \\
A & B & 0
\end{array}\right] \\
& =r\left[A A^{\dagger} B, B\right]+r\left[\begin{array}{cc}
0 & 0 \\
A & B
\end{array}\right]-r\left[\begin{array}{ccc}
0 & 0 & B \\
A & B & 0
\end{array}\right] \\
& =r\left[A A^{\dagger} B, B\right]-r(B) \\
& =r\left[A A^{\dagger} B,\left(I_{m}-A A^{\dagger}\right) B\right]-r(B) \\
& =r\left(A A^{\dagger} B\right)+r\left[\left(I_{m}-A A^{\dagger}\right) B\right]-r(B) \\
& =r[A, B]+r\left(A^{*} B\right)-r(A)-r(B) .
\end{aligned}
$$


Equality

$$
\min _{X} r\left(M-M\left[\begin{array}{c}
X \\
B^{\dagger}
\end{array}\right] M\right)=r[A, B]+r\left(A^{*} B\right)-r(A)-r(B)
$$

can be shown similarly. The equivalence of (a), (b) and (c) follows from (2.31).

TheOREm 2.5. Let $A \in \mathbb{C}^{m \times n}, B \in \mathbb{C}^{m \times k}, X \in \mathbb{C}^{k \times m}, Y \in \mathbb{C}^{n \times m}$, and let $M=[A, B]$. Then

$$
\min _{A^{-}, X} r\left(M-M\left[\begin{array}{c}
A^{-} \\
X
\end{array}\right] M\right)=\min _{Y, B^{-}} r\left(M-M\left[\begin{array}{c}
Y \\
B^{-}
\end{array}\right] M\right)=0 .
$$

Hence,
(a) There are $A^{-}$and $X$ such that $\left[\begin{array}{c}A^{-} \\ X\end{array}\right] \in\left\{M^{-}\right\}$.
(b) There are $Y$ and $B^{-}$and such that $\left[\begin{array}{c}Y \\ B^{-}\end{array}\right] \in\left\{M^{-}\right\}$.

Proof. Applying (1.25) and simplifying by elementary block matrix operations give us

$$
\text { (2.33) } \begin{aligned}
& \min _{X} r\left(M-M\left[\begin{array}{c}
A^{-} \\
X
\end{array}\right] M\right) \\
& =\min _{X} r\left(\left[0, B-A A^{-} B\right]-B X[A, B]\right) \\
& =r\left[B-A A^{-} B, B\right]+r\left[\begin{array}{cc}
0 & B-A A^{-} B \\
A & B
\end{array}\right]-r\left[\begin{array}{ccc}
0 & B-A A^{-} B & B \\
A & B & 0
\end{array}\right] \\
& =r\left[A A^{-} B, B\right]-r(B) \\
& =r\left[A A^{-} B,\left(I_{m}-A A^{-}\right) B\right]-r(B) \\
& =r\left(A A^{-} B\right)+r\left[\left(I_{m}-A A^{-}\right) B\right]-r(B) \\
& =r\left(A A^{-} B\right)+r[A, B]-r(A)-r(B) .
\end{aligned}
$$

Also from (1.28)

$$
\min _{A^{-}} r\left(A A^{-} B\right)=r(A)+r(B)-r[A, B] .
$$

Combining this with (2.33) leads to

$$
\min _{A^{-}, X} r\left(M-M\left[\begin{array}{c}
A^{-} \\
X
\end{array}\right] M\right)=0 .
$$

The second equality in (2.32) can be shown similarly. $\square$

Minimal rank formulas for column block matrices can be written out by symmetry. They are left for the reader. Some other topics on minimal ranks of generalized inverses of row block matrices can also be considered, for instance, to find the minimal ranks of

$$
[A, B]^{(i, \ldots, j)}-\left[\begin{array}{c}
\left(E_{B} A\right)^{(i, \ldots, j)} \\
\left(E_{A} B\right)^{(i, \ldots, j)}
\end{array}\right] \text { and }\left[A_{1}, \ldots, A_{p}\right]^{(i, \ldots, j)}-\left[\begin{array}{c}
A_{1}^{(i, \ldots, j)} \\
\vdots \\
A_{p}^{(i, \ldots, j)}
\end{array}\right]
$$


and then to use the minimal ranks to characterize the corresponding matrix equalities.

For $2 \times 2$ partitioned matrices, it is of interest to establish minimal rank formulas for the differences

$$
\left[\begin{array}{cc}
A & B \\
C & 0
\end{array}\right]^{(i, \ldots, j)}-\left[\begin{array}{cc}
A^{(i, \ldots, j)} & C^{(i, \ldots, j)} \\
B^{(i, \ldots, j)} & 0
\end{array}\right], \quad\left[\begin{array}{cc}
A & B \\
C & D
\end{array}\right]^{(i, \ldots, j)}-\left[\begin{array}{cc}
A^{(i, \ldots, j)} & C^{(i, \ldots, j)} \\
B^{(i, \ldots, j)} & D^{(i, \ldots, j)}
\end{array}\right] .
$$

It is expected that the rank formulas obtained can be used to characterize the corresponding equalities for generalized inverses of $2 \times 2$ partitioned matrices.

\section{REFERENCES}

[1] A. Ben-Israel and T.N.E. Greville. Generalized Inverses: Theory and Applications. 2nd Edn., Springer-Verlag, New York, 2003.

[2] S.L. Campbell and C.D. Meyer. Generalized Inverses of Linear Transformations. Corrected reprint of the 1979 original, Dover Publications, Inc., New York, 1991.

[3] G. Marsaglia and G.P.H. Styan. Equalities and inequalities for ranks of matrices. Linear and Multilinear Algebra, 2:269-292, 1974.

[4] L. Mihályffy. An alternative representation of the generalized inverse of partitioned matrices. Linear Algebra Appl., 4:95-100, 1971.

[5] C.R. Rao and S.K. Mitra. Generalized Inverse of Matrices and Its Applications. Wiley, New York, 1971.

[6] Y. Tian. Upper and lower bounds for ranks of matrix expressions using generalized inverses. Linear Algebra Appl., 355:187-214, 2002.

[7] Y. Tian. The minimal rank completion of a $3 \times 3$ partial block matrix. Linear and Multilinear Algebra, 50:125-131, 2002.

[8] Y. Tian. The maximal and minimal ranks of some expressions of generalized inverses of matrices. Southeast Asian Bull. Math., 25:745-755, 2002.

[9] Y. Tian. Rank equalities for block matrices and their Moore-Penrose inverses. Houston J. Math., 30:483-510, 2004.

[10] Y. Tian. More on maximal and minimal ranks of Schur complements with applications. Appl. Math. Comput., 152:675-692, 2004.

[11] H. Yanai. Some generalized forms of least squares $g$-inverse, minimum norm $g$-inverse, and Moore-Penrose inverse matrices. Comput. Statist. Data Anal., 10:251-260, 1990.

[12] S. Zlobec. An explicit form of the Moore-Penrose inverse of an arbitrary complex matrix. SIAM Review, 12:132-134, 1970. 